\title{
The challenges and possibilities of earthquake predictions using non-seismic precursors
}

\author{
A. Bhardwaj ${ }^{1,2, a}$, L. Sam ${ }^{1,2}$, and F. J. Martin-Torres ${ }^{1,2,3}$ \\ ${ }^{1}$ School of Geosciences, University of Aberdeen, Meston Building, King's College, Aberdeen \\ AB24 3UE, UK \\ ${ }^{2}$ Division of Space Technology, Department of Computer Science, Electrical and Space \\ Engineering, Luleå University of Technology, 97187 Luleå, Sweden \\ ${ }^{3}$ Instituto Andaluz de Ciencias de la Tierra (CSIC-UGR), Armilla 18100 Granada, Spain
}

Received 5 October 2020 / Accepted 7 October 2020

Published online 19 January 2021

\begin{abstract}
The catastrophic magnitude of life and monetary losses associated with earthquakes deserve serious attention and mitigation measures. However, in addition to the pre-earthquake and postearthquake alleviation actions, the scientific community indeed needs to reconsider the possibilities of earthquake predictions using nonseismic precursors. A significant number of studies in the recent decades have reported several possible earthquake precursors such as anomalies in electric field, magnetic field, gas/aerosol emissions, ionospheric signals, ground water level, land surface temperature, surface deformations, animal behaviour, thermal infrared signals, atmospheric gravity waves, and lightning. Such substantial number of scientific articles and reported anomalous signals cannot be overlooked without a thoughtful appraisal. Here, we provide an opinion on the way forward for earthquake prediction in terms of challenges and possibilities while using non-seismic precursors. A general point of concern is the widely varying arrival times and the amplitudes of the anomalies, putting a question mark on their universal applicability as earthquake markers. However, a unifying concept which does not only define the physical basis of either all or most of these anomalies but which also streamlines their characterisation procedure must be the focus of future earthquake precursory research. Advancements in developing the adaptable instrumentation for in-situ observations of the claimed non-seismic precursors must be the next step and the satellite observations should not be taken as a replacement for field-based research. We support the need to standardise the precursor detection techniques and to employ a global-scale monitoring system for making any possible earthquake predictions reliable.
\end{abstract}

a e-mail: anshuman.bhardwaj@abdn.ac.uk 


\section{Meaning of prediction}

The purpose of this opinion piece is not to repeat the review of proposed non-seismic earthquake precursors which have already been well-described by several articles [1-7]. We intend to discuss the possibilities of advancements and improvements in earthquake prediction while using non-seismic precursors. However, before starting with the scientific discussion on the already well-debated topic of earthquake prediction, one needs to first revisit the linguistics of the word "prediction". A basic definition of "prediction" as provided by the Cambridge Advanced Learner's Dictionary and Thesaurus (https://dictionary.cambridge.org) refers to it as a statement that one makes about future events based on one's perceptions. These perceptions might be based on logical reasoning or they might be completely intuitive, and thus either they may accurately define the future events or they may go way off-target [8].

The risks or uncertainties are inherent to any kind of prediction or forecasting [9] but does this mean that one should stop speculating and making first-hand predictions within scientific, social science, political, and philosophical domains and would not doing that contradict the very first step of research methodology, i.e., developing hypothesis, before testing it to provide justifiable conclusions? Thus, while we see that the idea of "prediction" is not particularly antagonistic for the people working in fields such as hydrology (e.g., [10]), climatology (e.g., [11]), statistics (e.g., [12]), material science (e.g., $[13,14]$ ), biomedicine (e.g., $[15,16])$, economics (e.g., [17]), psychology (e.g., [8]), political science [18], and literature (e.g., [19]), when it comes to seismology, the concept of prediction can be unsettling for many (e.g., [20-23]). As a critic of the earthquake prediction attempts, Geller [21] starts with an opening statement citing Franks [24], where Felix Franks relates "alleged breakthroughs that may perhaps lead to a cure for cancer, or more exact prediction of earthquakes, or greatly improved crop yields" to the alluring possibilities of exposure and publicity which can be hard for scientists to resist. We will not comment on the audacity of this statement and we acknowledge that when the statement was made, i.e., in 1981, the mentioned scientific advancements might have seemed like a distant dream. However, today we certainly know that the past three decades have seen tremendous medicinal, biotechnological, technological, and computing advances leading to exponential increase in the number of cancer survivors [25] as well as in the global cereal production [26]. A more matured viewpoint on earthquake prediction was put forth by C. F. Richter in his acceptance speech of the 1976 Harry Fielding Reid Medal of the Seismological Society of America, wherein although he deemed most predictions to be little more than attention seeking attempts, he also stated that such attempts based on practicality, appropriate use of precise information, and understanding of the intrinsic complications might be useful $[27,28]$. Regarding earthquake prediction, we would like to start this discussion with the statement that instead of straightaway deeming prediction efforts as overenthusiasm or a publicity stunt, they should be considered as cautious optimism based on continuously improving seismological and non-seismic precursor measuring instruments and techniques, in order to facilitate an open mind for their further unbiased scrutiny. However, we also understand that the most important and initial component of any prediction effort is not necessarily to have good instruments or techniques but to possess an in-depth understanding of the physical processes that take place prior to major earthquakes. Once this understanding is achieved with a definite degree of certainty, the selection of the instruments and methods becomes easier and reliable.

Now, if we consider the word "prediction" in terms of strict seismological significance, understandably being majorly off-target is not an option since not only significant number of lives are at stake in case of inability to predict, but also in case of a false alarm, substantial wasted mitigation resources and measures can prove to 
be costly and economically unviable. Nevertheless, a forecasting of an earthquake can prompt several easy and cost-effective measures instead of complete evacuation, even if the forecast lacks $100 \%$ reliability $[29,30]$. The documentations on the attempts for earthquake predictions show a timeline of more than a century [21] but focused efforts in this direction primarily started during 1960s in Japan in collaboration with foreign institutes and the optimism was so high in the beginning that these initiatives found positive research coverage (e.g., [31]). However, even during the early days of earthquake prediction in Japan, the approach was appropriately cautious and emphasised on the urgency of obtaining fundamental data pertaining to geodesy, crustal deformation, seismicity, fault mechanisms, and geomagnetic-geoelectric signals for possible prediction rather than on rushing for immediate forecasting [31]. In the succeeding years, United States National Research Council's Panel on Earthquake Prediction of the Committee on Seismology (PEPCS) considered critical technical evaluation of earthquake prediction as the necessary first step and acknowledged the great potential that precursory research encompassed in saving lives [32]. In addition, this panel also proposed preliminary premises for prediction research in terms of their ability to provide the expected magnitude range within the specified geographical boundaries in the expected time interval with sufficient enough precision to facilitate assessment of the eventual success or failure of the prediction. In the same year, in his Presidential Address to the Seismological Society of America, delivered in Edmonton, Alberta, Allen [33] suggested six essential elements of earthquake prediction: (i) time window, (ii) space window, (iii) magnitude window, (iv) degree of confidence, (v) chances of the unpredictable earthquakes as random events, and (vi) easily accessible and understandable documentation for further assessments. However, prediction in these initial years strictly corresponded to studying recursive geophysical phenomena until Vere-Jones [34] suggested incorporation of statistical robustness, and certainty and risk assessment which received more attention in the following years [35]. In particular, Vere-Jones [34] questioned the premises of a prediction in terms of exact time, space, and magnitude as such precision seemed impractical and suggested introduction of error limits and occurrence probability which could eventually be called risk formulation for prediction failure. Vere-Jones [34] also explained advantages of such risk formulations as they could make the predictions less panicking for the public, in addition to facilitating rule-based decision making for economic viability of pre-earthquake mitigations. Of course, then the next question arises on the precision and methods of such probabilistic risk formulations for prediction failures and in a follow-up paper, Vere-Jones [36] discussed this in detail. Vere-Jones [36] stated that probabilistic risk formulations could be best developed from models which consider a time-varying conditional probability per unit time, area or volume, and magnitude interval for every point in a given spatial domain; a concept of time-space-magnitude domain earlier approved by the United Nations Educational, Scientific and Cultural Organisation (UNESCO) and the International Association of Seismology and Physics of the Earth's Interior (IASPEI) [37]. Vere-Jones [36] further provided four essential seismic parameters (epicentre location, depth, origin time, and magnitude) and six accessory seismic parameters (foreshocks, aftershocks, focal mechanism, seismic moment, energy release, and earthquake intensity) to ensure a meaningful statistical analysis. Understandably, Vere-Jones [36] did not discuss nonseismic earthquake parameters since such precursors have been majorly reported in the past two decades which have seen significant Earth observation and computing advancements.

Following the PEPCS recommendations on the scale and spatiotemporal premises of earthquake prediction [32] and subsequent addition of probability to that definition 


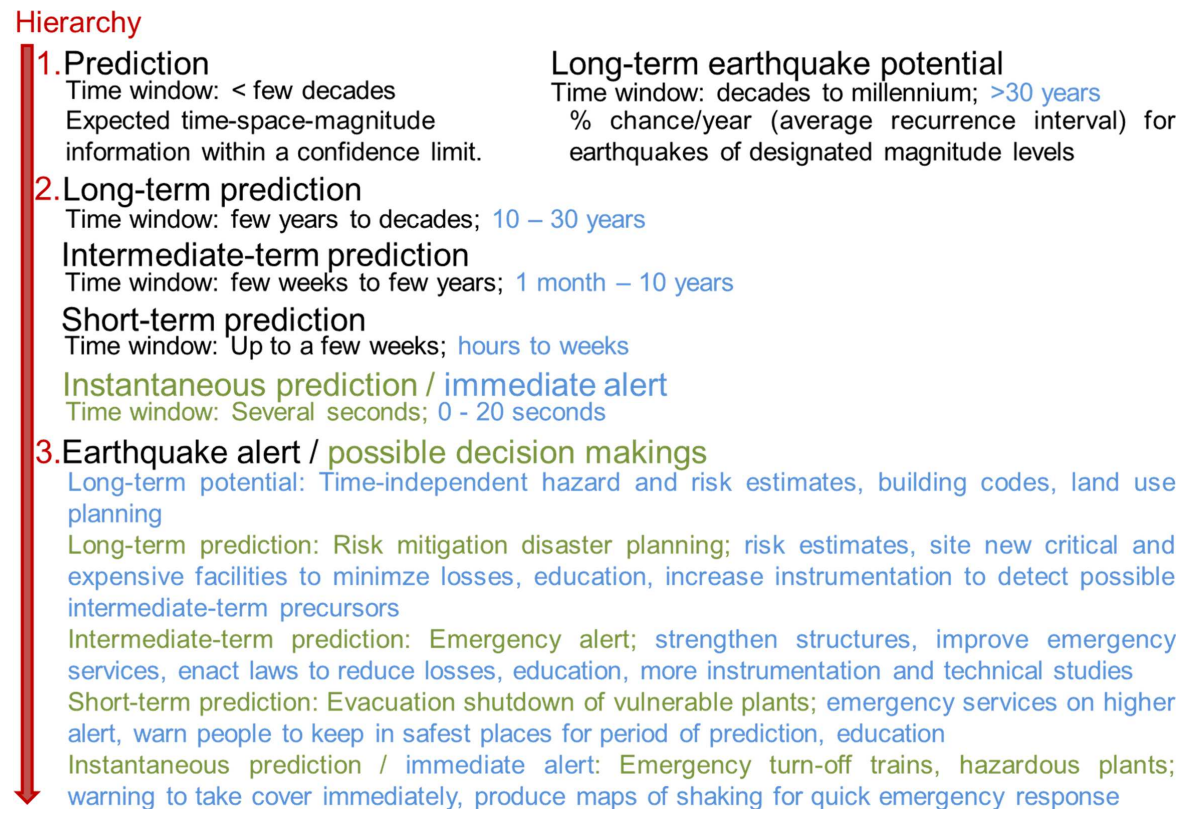

Fig. 1. Temporal hierarchy in earthquake prediction. The texts in black show the levels proposed by Wallace et al. [38] with subsequent additions by Snieder and Van Eck [39] and Sykes et al. [40] highlighted by the green and blue texts, respectively.

[34], Wallace et al. [38] focused on the time element of a prediction to further refine the basic definition of prediction (Fig. 1). Wallace et al. [38] proposed three hierarchical steps in earthquake prediction based on the time domain of prediction as shown in Figure 1. They put prediction and long-term earthquake potential at the top of the ranks, followed by short-, intermediate-, and long-term predictions at the second tier, and earthquake alert at the lowest level. They further expected future sub-divisional additions in these levels with an overall undisturbed framework. Snieder and Van Eck [39] placed instantaneous prediction corresponding to a preparedness time of several seconds in the middle level and also added possible decision-making recommendations in the hierarchy for all kinds of predictions detailed in the middle tier (Fig. 1). Sykes et al. [40] further provided several modifications to this classification by providing absolute time values to the time element, by adding immediate alert level which is equivalent to instantaneous prediction of Snieder and Van Eck [39], and by adding more decision making suggestions, even for the long-term earthquake potential (Fig. 1). Such suggestions [34,36,38-40], on incorporating statistical and time constraints in earthquake predictions certainly cleared some vagueness on prediction in seismic sense. Kagan [41] further supported the idea by Vere-Jones [34,36], that earthquake prediction must be defined and evaluated in statistical context and questioned the existing definitions of earthquake prediction. Kagan [41] did not approve of the prevalent time-space-magnitude domain definition of earthquake prediction and deemed such prediction improbable due to the continuum and fractal nature of the statistical distributions of earthquake. In addition, Kagan [41] also highlighted the absence of robust acceptable criteria to validate an earthquake prediction as the commonly used failure-to-predict versus false-alarm criterion can fail due to operational trivial alarm declaration strategy for the entire region and is also ineffective to distinguish a method with real predictive ability from chance coincidences, i.e., the null hypothesis is undefined. In addition, the rigorous testing of prediction attempts also 
get difficult due to an insufficient number of predicted events, or prediction uncertainties, or a prediction time window too large for the testing to be viable [41]. Due to the scale-invariance of seismicity, instead of temporal classification based on actual time element as suggested by others [38-40], Kagan [41] proposed temporal classification based on physical scales associated with the elastic wave propagation and earthquake rupture (seconds to minutes), and the velocity of tectonic deformation (decades to millennia). However, the latter tectonic deformation-based decades to millennia prediction scale is again too large to validate and contradicts Kagan's [41] own arguments on the inability to assess previously suggested classifications [38,39]. Nevertheless, Kagan [41] provided several prediction characteristics out of which, predictive ratio (number of successfully predicted events relative to forecast using the null hypothesis) and precision (difference between prediction errors and forecast using the null hypothesis) can directly be used to statistically assess the prediction success. In this regard, Molchan's suggestions [42-44] are worth a mention here as he suggested inclusion of specific social, economic, and geophysical goals too while performing the whole exercise of earthquake prediction. Molchan's suggestions [44] highlight the fact that ultimately the prediction efforts and results are going to be effective only when they foresee the subsequent decision-making issues and incorporate the relevant socioeconomic considerations. Molchan [42-44] also presented the Schematic Errors Diagram or Prediction Errors Diagram to solve the problem of comparing prediction methods for stationary point events or a sequence of strong earthquakes in a region by using two prediction parameters: (i) the rate of prediction failures, i.e., the number of missed events divided by the total number of events in a given time interval, and (ii) the rate of time alarms, i.e., the total time of alarms divided by total time interval.

In the following years, the seismic meaning of prediction further evolved. Jackson [29] cleared the ambiguity between prediction and forecast. He defined earthquake forecasting as stipulating the long-term probability of earthquakes in timespace-magnitude domain with incorporation of modest time-dependence. In contrast, Jackson [29] defined earthquake prediction in a more rigorous sense as specifying special conditions that make the immediate probability much higher than usual, and high enough to describe what is unusual. Joffe et al. [28] after interviewing several Earth Science researchers concluded that the scientific community is more receptive of the long-term forecasting instead of short-term predictions. However, such safe attitude of avoiding the topic of short-term prediction is not an option since these near-future predictions are the ones which are more crucial in reducing the vulnerability and risks. Jordan [45] defined intrinsic predictability as the degree of prediction based on the precursory behaviour of an active fault system. He further defined a scientific prediction as a testable hypothesis (usually probabilistic) in time-space-magnitude domain, and distinguished it from a useful prediction which signifies the early warning of potentially destructive fault rupture with enough accuracy in space and time to warrant mitigation actions for communities. The International Commission on Earthquake Forecasting (ICEF), set up in the aftermath of the L'Aquila earthquake presented a different view while distinguishing prediction from forecast to the one proposed by Jackson [29]. According to ICEF, a prediction is a deterministic statement that a future earthquake will or will not occur in a particular time-space-magnitude range, whereas a forecast gives a probability (in the range of $0-1$ ) that such an event will occur $[46,47]$.

From the above-mentioned compilation and discussion of the seismic meaning of prediction, we have several inferences. Over the past decades, the definition of earthquake prediction has continuously evolved and in addition to initial time-spacemagnitude information, prediction now encompasses probability, statistical testing, and confidence level for issuing alerts. Thus, associating the word prediction with an 
expectation of $100 \%$ reliability is not only unfair but also obviously unphysical. The reason is that not every stress build-up in the rock column leads to a catastrophic rupture, i.e. an earthquake [3]; tectonic stresses can also be relieved by other processes, most commonly slow sliding, giving rise to "silent earthquakes" [45,48]. Thus, to demand $100 \%$ reliability is unfair to the researchers who are interested in at least trying in that direction. It is also demoralising for practitioners who need to assess the immediacy of the danger. In case of non-seismic precursors which are the focus of this article, the targeted predictions are primarily of short- and intermediate-terms as the observed precursors have been reported days to weeks prior to the earthquakes. Due to the short-term predictability offered by such non-seismic precursors, they are more prone to creating public panic or economic constraints on mitigation measures. This further warrants a need to perform robust scrutiny of such precursors before considering them for possible predictions. In the next section, we take a look at such non-seismic precursors explained in the published literature.

\section{Non-seismic precursors and prediction challenges}

There are several non-seismic precursors mentioned in literature and instead of repeating the related details, we have compiled several of the most commonly reported non-seismic precursors in Table 1 along with the relevant articles so that the interested readers can get in-depth background information on them. As mentioned, the non-seismic precursors are primarily useful to try and achieve short-term predictability, which is otherwise even more difficult to accomplish using conventional mechanical or seismic approaches due to their inability to predict the expected time of attainment of critical stress levels using contemporary seismic or geodetic instruments [49]. Non-seismic precursors supposedly have lower critical stress levels making them appear days or weeks before earthquakes and are believed to be the by-products of the same stress build-up that leads to earthquakes [49]. Their classification within short- or intermediate-term predictability is based on the observations reported in the literature (References in Table 1) where the studies have reported their appearances majorly hours to months before the earthquakes.

Such precursors have been reported to appear in wide range of electro-magnetic (EM) spectrum starting from thermal infrared (TIR) [1,60,61,77] and going up to ultra-low frequencies (ULF) $[90,91]$. This can explain the difference in arrival or detection times of these precursors before an earthquake but does not necessarily support the idea of different generation mechanisms for them as proposed earlier (e.g., [49]). In fact, the possible common source of origin for all the reported precursors mentioned in Table 1 also signifies that there has to be one stress-related physical explanation for if not all then at least majority of such precursors (e.g., [92]). The main arguments in the papers (e.g., [49]) refuting the lab-based observations such as generation of positive holes ( $p$-holes) in igneous rock under local stress (e.g., [92,93]), hypotheses based on electro-kinetic effects or pressure stimulated current (PSC) effects [52,94], and models of micro-cracking (e.g., $[95,96])$ is the unavailability of enough field evidences to prove them in natural environment. This argument is certainly valid in the present circumstances but then the most genuine question in the backdrop of such refutation is that are we actually technically and financially well-equipped to gather such field evidences for different ranges of earthquake magnitudes, focal depths, and fault types from several places around the globe? The answer to this question is a simple "no" because of the prevalent perpetual pessimism surrounding earthquake predictions which has quenched the needed level of federal funding and support. If we can find field evidences for even certain magnitude ranges, fault types, and focal 
Table 1. Commonly reported non-seismic precursors. Top seven of these represent aboveground precursors (up to ionosphere), followed by four precursors at the surface-level, and the bottom-most represents below-ground observations.

\begin{tabular}{ll}
\hline Precursor & References \\
\hline Electric field & {$[50-52]$} \\
Magnetic field & {$[53,54]$} \\
Electromagnetic anomaly & {$[55,56]$} \\
Gas/aerosol emissions & {$[57-59]$} \\
Outgoing longwave radiation (OLR) & {$[60-62]$} \\
Ionospheric anomaly & {$[63-67]$} \\
Air temperature anomaly & {$[68,69]$} \\
Geochemical changes & {$[70,71]$} \\
Surface deformations & {$[72-76]$} \\
Land surface temperature (LST) anomaly & {$[1,77-80]$} \\
Unusual animal behaviour & {$[81-85]$} \\
Groundwater levels and chemistry & {$[4,86-89]$} \\
\hline
\end{tabular}

depths in sufficient number of cases to perform generalised characterisations, it can certainly change the complete scenario.

The reported time of pre-earthquake detection of the non-seismic signals vary considerably. For TIR and land surface temperature (LST) anomalies, the reported arrival of anomalous signals varies from days to $\sim 3$ weeks [1]. Unusual animal behaviours have been observed either on the earthquake dates or since few days earlier to the onset [81]. The arrival of hydro-geochemical anomalies has been reported one to ten weeks prior to earthquakes [97]. Electromagnetic and ionospheric anomalies have been observed hours to a few days before the earthquakes [55]. Surface deformations have been detected days to years before the earthquakes [75]. Thus, the temporal span of arrival of such anomalous signals may vary from minutes to years before the earthquakes and makes the prospect of prediction even more difficult. In addition, the amplitudes of such signals also vary substantially [1]. Furthermore, any correlation between the amplitudes of anomalies and the magnitudes of corresponding earthquakes is considerably vague. Several of the research articles for each of the precursors mentioned in Table 1 have displayed sufficient methodological and statistical robustness to characterise the anomalies but the application of different analytical approaches for the same earthquake have often resulted in different appearance time and amplitudes for the same precursor. Such randomness poses another constraint on the universal applicability of these precursors for issuing earthquake alerts. This signifies a need to not just unifying the physical basis for such precursors but also to standardise the measuring and statistical approaches after thoughtful considerations. These methodological approaches must be proven effective in accounting for natural variability (temperature, humidity, lightening, solar fluxes, and cloudiness) due to topography, climate, and weather to characterise the anomalies. Ideally the approach should be independent of the areal or temporal scales of observations to allow for a wider applicability but if that is difficult to attain, then it must at least define the observational conditions pertaining to spatiotemporal boundaries for effective detection. Obviously long-term localised observations improve the statistical robustness but a conclusive convention on such spatiotemporal boundaries needs to be reached.

Considering the variability in the type of precursors (Tab. 1), we recommend to focus on the instrumentation aspect which has taken the backseat in the recent decades while we believe that it should be the prime necessity if we have to attain the prediction goals. With the advances in satellite remote sensing and computing, 
the majority of the research on earthquake prediction has shifted its focus to improving the data analytics. We see the lack of enough funding for precursory research also as a reason for such research trends. As mentioned above, the field instrumental records are irreplaceable and the most crucial factor to define the course of earthquake prediction research. However, in the present scenario and with funding crisis, there is a need to focus on two main factors while deciding instrumentation development: (1) the instrumentation has to be self-sustaining in harsh environments (to ensure data continuity in all weather and terrain scenarios), capable of real-time data relay (to allow real-time data backup and analyses), and must be inexpensive (to facilitate its wide reach and applicability to any researcher); and (2) the instrumentation should be holistic (a multi-sensor approach) in the way that it should be capable of detecting if not all then at least majority of the precursors mentioned in Table 1 . The next step should be to strategically place such instruments at different locations around the globe so that they can cover different fault and earthquake types. In this regard, a proof-of-concept prototype has been developed at the Luleå University of Technology, Sweden [98]. The instrument prototype demonstrated self-sustainability throughout the Arctic winter of 2017-18 and provided data from all the installed sensors in real-time. The instrument is equipped with different modules of temperature and relative humidity sensors, magnetometer, geophone, conductivity meter, and infrasonic sensors at different levels within and above ground. It runs on solar and wind power and consists of commercial off-the-shelf (COTS) components which provide structural flexibility in addition to bringing down the developmental and operational costs of the instrument considerably (https://atmospheres.research.1tu.se/pages/projects/s3me2.php). Details on this instrument are subject of another scientific article but its mention here was needed to highlight that such instrumentation can be developed at low costs with high productivity to approve or disapprove of several of the precursors mentioned in Table 1 and can further give a new direction to the operational earthquake prediction efforts. This instrument is developed particularly to detect majority of the precursory signals mentioned in Table 1 and in alignment with the unifying solid-state concept proposed by Freund [92]. Presently the instrument is in the testing phase and unless we install it on the field and start receiving continuous data, we cannot comment on the vital analytical shifts that it is expected to provide. However, we firmly believe that since such instruments can gather relevant data on several parameters and with significantly higher frequency than the satellites, they must improve our understanding of the ongoing geophysical processes in seismic zones. One important aspect in view of more of such instruments in future will be to encourage mutual data sharing amongst the research communities and development of robust anomaly characterisation routines which are statistically sound and which can make better use of such high-frequency observations. Another way forward should be the comparison studies of such on-ground sensors with the remote sensors to identify the extent of dissimilarities or biases and how it can further affect the anomaly characterisation while solely depending on the satellite observations.

We probably do not need multiple physical models to explain the reported anomalies in Table 1 as they can majorly be described through a unified model. Based on continuous research spanning several decades, Friedemann Freund [92,99-101] proposed a "peroxy defects" mechanism through a holistic consideration of lithochemistry, semiconductor physics, and rock physics and explained how rupture of the peroxy bonds (PHPs) and the subsequent activation of $p$-holes in rocks subjected to ever-increasing tectonic stresses prior to any major seismic activity can induce multitude of electromagnetic precursors. We can understand it by taking a look at three different kinds of anomalies and how they can be explained by peroxy defects model: (i) physical signal (e.g., LST anomaly), (ii) chemical signal (e.g., groundwater 
chemistry), and (iii) biological signal (e.g., unusual animal behaviour). If we first consider the reported LST anomalies before earthquakes, they are essentially midinfrared (MIR) luminescence anomalies observed via thermal remote sensors. Such unusual MIR luminescence is essentially a result of electromagnetic perturbations and can certainly be explained by the induced charge flows through the rocks [92]. However, what is more interesting to mention here is the possible ways in which the peroxy defects model can explain case-specific LST observations too. For example, Bhardwaj et al. [77] observed higher LST anomalies for the snow-covered mountains for the three highest magnitude alpine earthquakes (Nepal, Chile, and Afghanistan) of 2015. These findings corroborated several previous observations $[102,103]$ for the M6.3 L'Aquila earthquake where the LST anomaly was reported to be concentrated on the mountain peaks with essentially no anomalous detections on the valley floor consisting of active faults and the earthquake epicentre. The mountains in both the studies $[77,103]$ are largely composed of limestone which can display high exhalation rates for $\mathrm{CO}_{2}$ [103]. Such $\mathrm{CO}_{2}$ and $\mathrm{Rn}$ release can be facilitated by micro-fracturing induced by stress-induced peroxy defects and ion propagations [92]. The released $\mathrm{CO}_{2}$ is a greenhouse gas and can further act as a Rn carrier gas, thus contributing to the thermal anomaly at the mountain peaks [104]. The peroxy defects mechanism can also explain the relatively enhanced signals in snowpack or ice layers as the $p$-holes can propagate through ices containing traces of $\mathrm{H}_{2} \mathrm{O}_{2}$ [105] and nearly all natural ices as a result of UV irradiation at high alpine peaks are expected to possess traces of $\mathrm{H}_{2} \mathrm{O}_{2}$. In the similar manner as above, the reported changes in groundwater chemistry ( $\mathrm{pH}$, total dissolved solid (TDS), $\mathrm{Rn}, \mathrm{CO}_{2}, \mathrm{He}, \mathrm{CH}_{4}, \mathrm{H}_{2}, \mathrm{Ar}$, and $\mathrm{N}_{2}$ ) can also be explained by the peroxy defects mechanism governing the generation and propagation of $p$-holes through the rock columns, further inducing electrochemical processes at the rock-water interface and building up an electric potential [4]. These $p$-holes are highly oxidising and they oxidise $\mathrm{H}_{2} \mathrm{O}$ to $\mathrm{H}_{2} \mathrm{O}_{2}$ and a chain of oxidation reactions sets up to give rise to oxygen, hydrogen, and hydroxyl radicals which are strong enough to display all the reported anomalous physicochemical signals within the groundwater prior to an earthquake. However, the most interesting aspect of peroxy defects mechanism as a unifying concept is the possibility that it can actually explain the observed biological anomalous signals such as unusual animal behaviour prior to an earthquake $[82,106]$. Several animal species are known to be perceptive to the electromagnetic wavelengths which are extreme and beyond human receptors [107]. The flow of p-holes produces electromagnetic perturbations in a wide range of the spectrum to which such animals can be repulsive and they respond unusually.

As mentioned above, the satellite-based observations of pre-earthquake anomalies clearly display high degree of heterogeneity with significant predisposition on the factors such as spatial domain of the observations, temporal span of the measurements, magnitude of the earthquakes, statistical approach for anomaly characterisation, ambient noise, and electromagnetic spectrum of the observations. In fact, the cited literature here widely suggests that more volume of temporal data one uses, the smaller becomes the amplitude of the anomalies which at first seems quite puzzling but is comprehensible upon a deeper thinking. For example, if we consider the M7.6 Gujarat (India) earthquake of 26 January 2001, we will find several studies reporting MIR and TIR anomalies [e.g., [59,108,109]] while using the data from relatively shorter temporal spans (either few days surrounding the earthquake or the LST difference between the year of earthquake and another year without an earthquake). These studies report an anomaly amplitude of $\sim 3-7^{\circ} \mathrm{C}$ appearing $\sim 1-2$ weeks before the earthquake. However, for the same earthquake, when Blackett et al. [110] analysed data of six continuous years (2001-2006), they reported significantly smaller magnitude of anomaly that fell well within the inter-annual anomaly envelope of standard deviations. While this seems discouraging in terms of earthquake predictability, 
a positive inference can be the fact that inclusion of multi-year observations actually improves the statistical robustness of the anomaly characterisation method by accounting for the natural background noise signals which may dramatically vary during a particular year depending on the short-term weather conditions. This natural variability also indicates towards another possibility that has actually been observed in certain cases; the variations in in-situ observations of anomalies by very closely situated monitoring stations. Such variability can be explained either by the variations in local background environmental conditions based on topographic variables or by the lithological variations where one station might be operating directly over the bedrock (e.g., limestone) while the other station although closer in terms of aerial distance might be located on valley floor or sedimentary soil. In this regard, we again see the peroxy defects mechanism as a unifying method to overcome such observational as well analytical heterogeneity. However, we understand that in natural environment, displaying and proving a unifying concept such as the peroxy defects mechanism through acquisition of relevant data is still a challenge but its physical understanding can be a good starting point to design the relevant field instrumentations and statistical approaches for anomaly characterisation.

\section{Optimisation and way ahead}

The entire approach to earthquake prediction using non-seismic precursors needs optimisation in terms of cost-effective instrumentation, and standardisation of statistical methods and research designs. The statistical tools to quantify anomalies are existent but their optimal use and effectiveness largely depend on univocal and quantifiable characterisation of the hypothesis to be tested. In the early 1990s, Molchan through several of his papers $[42,43,111]$ stressed upon the need of optimisation of short-term predictions by focusing on specific social, economic, and geophysical goals. However, in the past two decades, the scenario has although significantly improved due to availability of a variety of remote sensing data, the optimisation and standardisation efforts have been minimal leading to even more randomisation in earthquake precursory research. New "precursors" have evolved in the recent decades thanks to wide spatial coverages provided by Earth observation satellites and the focus has shifted to observing and reporting them instead of streamlining the anomaly characterisation routines for an effective prediction. For example, as we mentioned in Section 2, while performing a literature survey, we do not just find a multitude of precursors reported in the recent years (Tab. 1) but we also find a significant randomness in their spatiotemporal occurrences and amplitudes.

In relation to optimising the prediction process, the next step should be to rethink on the proper way of discussing and representing the prediction. Associating any new method of earthquake prediction first with inherent uncertainty is a better start than directly discussing its reliability. Provision of uncertainty provides some breathing space to any novel prediction attempt and does not discourage the progress of predictive research in its very beginning. A very basic problem hindering the prediction attempts significantly has resulted from the arguments that such predictions can cause public panic and if they are not accurate, they can lead to significant monetary loses in terms of mitigation measures. However, a supposedly simple and starting point for such prediction attempts should be to select the study area which is secluded from any civilisation. This also provides the opportunity of achieving a suppressed background noise which otherwise can give mixed signals to the measuring instruments. This will be the best way to test a novel approach without causing any unwanted turmoil. Several such sites which are seismically very active, pristine, and signify wide wilderness can be in Iceland, and Himalayan and Andean mountains. 
After the time of the predicted event passes by, the next stage of discussing the reliability of the new technique in terms of time-space-magnitude domain within the uncertainty bounds should come into picture. At this stage, even if a certain level of predictability is achieved within the proposed uncertainty in the spatiotemporal domain, that will help in further narrowing down the uncertainty limits. The approach can be tested for several more earthquakes before applying it for a habitation zone. As we have discussed in Section 2, involving multi-year observations are helpful in differentiating the actual anomalies from the background noises with higher statistical confidence and we believe that continuous 5-10 years of in-situ observations should be a baseline to further achieve the results with robust analyses.

The present need is to implement the basics of seismic research to non-seismic precursory research too, i.e., to encourage the development of adaptable instrumentation which can self-sustain in harshest of the Earth environments and can provide in-situ observations of non-seismic precursors in real-time. Although the science of earthquake prediction is in the picture since past several decades, the long list of failures does not allow it to be called an established science. In such scenarios, the satellite observations must not be taken as a replacement for field-based research and should only be used as accessory resources unless a needed level of robustness is achieved. We of course acknowledge the contribution of satellite data in non-seismic precursory research but what we are reiterating here is the basic fact that remote sensing must be complemented with robust in-situ observations. However, our bigger concern is not just the growing cases of a complete reliance on satellite data but the shift in the focus of the non-seismic precursory research to somehow find the "anomalies" without giving in-depth considerations on their reliability. We support the need to standardise the precursor detection techniques and to employ a global-scale monitoring system for making the earthquake predictions reliable. This will undoubtedly need substantial funding support but the most important thing as of now is to incorporate a higher level of practicality and robustness in our entire approach towards earthquake precursory research. A zero-success rate of earthquake predictions would have been certainly demotivating but the success stories [112], although very few in number, are motivating enough for us to realise that there might be a possibility and a pessimistic approach should not be opted as the easier option.

The authors are grateful to the editors and the reviewers for their constructive and useful suggestions in improving the quality of the paper.

Open Access This is an open access article distributed under the terms of the Creative Commons Attribution License (https://creativecommons.org/licenses/by/4.0/), which permits unrestricted use, distribution, and reproduction in any medium, provided the original work is properly cited.

Publisher's Note The EPJ Publishers remain neutral with regard to jurisdictional claims in published maps and institutional affiliations.

\section{References}

1. A. Bhardwaj, S. Singh, L. Sam, P.K. Joshi, A. Bhardwaj, F.J. Martín-Torres, R. Kumar, Int. J. Appl. Earth Obs. 63, 158 (2017)

2. R.D. Cicerone, J.E. Ebel, J. Britton, Tectonophysics 476, 371 (2009)

3. F. Freund, J. Asian Earth Sci. 41, 383 (2011)

4. S.R. Paudel, S.P. Banjara, A. Wagle, F.T. Freund, J. Seismol. 1 (2018) 
5. A.K. Saraf, V. Rawat, S. Choudhury, S. Dasgupta, J. Das, Int. J. Appl. Earth Obs. 11, 373 (2009)

6. M. Verma, B.K. Bansal, J. Asian Earth Sci. 54, 1 (2012)

7. J. Zhong-Hu, J. Zhao, X. Shan, Nat. Hazard. Earth Sys. 18, 1013 (2018)

8. D. Kahneman, A. Tversky, Psychol. Rev. 80, 237 (1973)

9. J.S. Chadha, Nat. Inst. Econ. Rev. 239, F4 (2017)

10. D. Demeritt, H. Cloke, F. Pappenberger, J. Thielen, J. Bartholmes, M.H. Ramos, Environ. Hazards-UK. 7, 115 (2007)

11. J. Slingo, T. Palmer, Phil. Trans. R. Soc. A 369, 4751 (2011)

12. P. Spirtes, C.N. Glymour, R. Scheines, Causation, prediction, and search (MIT press, London, England, 2000)

13. G. Frohnsdorff, L.W. Masters, The Meaning of Durability and Durability Prediction, in Durability of Building Materials and Components, edited by P.J. Sereda, G.G. Litvan (American Society for Testing and Materials, Ottawa, Canada, 1980), pp. 17-30

14. A.Y. Liu, M.L. Cohen, Science 245, 841 (1989)

15. P.M. Ridker, C.H. Hennekens, J.E. Buring, N. Rifai, New Engl. J. Med. 342, 836 (2000)

16. P.W. Wilson, R.B. D'Agostino, D. Levy, A.M. Belanger, H. Silbershatz, W.B. Kannel, Circulation 97, 1837 (1998)

17. C.F. Manski, Econ. Lett. 91, 425 (2006)

18. A.G. Cuzán, C. M. Bundrick, Polit. Anal. 17, 333 (2009)

19. F. Smith, Elem. Engl. 52, 305 (1975)

20. W.H. Campbell, Eos, Trans. Am. Geophys. Union 79, 463 (1998)

21. R.J. Geller, Geophys. J. Int. 131, 425 (1997)

22. R.J. Geller, D.D. Jackson, Y.Y. Kagan, F. Mulargia, Science 275, 1616 (1997)

23. A. Saegusa, Nature 397, 284 (1999)

24. F. Franks, in Polywater (MIT Press, 1981), pp. 126-127

25. C.E. DeSantis, C.C. Lin, A.B. Mariotto, R.L. Siegel, K.D. Stein, J.L. Kramer, R. Alteri, A.S. Robbins, A. Jemal, CA-Cancer J. Clin. 64, 252 (2014)

26. D. Tilman, K.G. Cassman, P.A. Matson, R. Naylor, S. Polasky, Nature 418, 671 (2002)

27. B.F. Howell, C.F. Richter, Bull. Seismol. Soc. Am. 67, 1243 (1977)

28. H. Joffe, T. Rossetto, C. Bradley, C. O'Connor, Disasters 42, 81 (2018)

29. D.D. Jackson, Earthquake prediction and forecasting, in The State of the Planet: Frontiers and Challenges in Geophysics, Geophysical Monograph (IUGG, 2004), Vol. 19

30. D.L. Turcotte, Ann. Rev. Earth Pl. Sc. 19, 263 (1991)

31. T. Rikitake, Earth-Sci. Rev. 4, 245 (1968)

32. National Research Council, Predicting earthquakes: a scientific and technical evaluation, with implications for society (National Academy of Sciences, Washington, 1976)

33. C.R. Allen, Bull. Seismol. Soc. Am. 66, 2069 (1976)

34. D. Vere-Jones, J. Phys. Earth 26, 129 (1978)

35. Y.Y. Kagan, L. Knopoff, Science 236, 1563 (1987)

36. D. Vere-Jones, Int. J. Forecasting 11, 503(1995)

37. IUGG, Code of practice of earthquake prediction, IUGG Chronicle No 165 (1984)

38. R.E. Wallace, J.F. Davis, K.C. McNally, Bull. Seismol. Soc. Am. 74, 1819 (1984)

39. R. Snieder, T. Van Eck, Geol. Rundsch. 86, 446 (1997)

40. L.R. Sykes, B.E. Shaw, C.H. Scholz, Pure Appl. Geophys. 155, 207 (1999)

41. Y.Y. Kagan, Geophys. J. Int. 131, 505 (1997)

42. G.M. Molchan, Phys. Earth Planet. In. 61, 84 (1990)

43. G.M. Molchan, Tectonophysics 193, 267 (1991)

44. G.M. Molchan, Pure Appl. Geophys. 149, 233 (1997)

45. T.H. Jordan, Seismol. Res. Lett. 77, 3 (2006)

46. T. Jordan, Y. Chen, P. Gasparini, R. Madariaga, I. Main, W. Marzocchi, G. Papadopoulos, G. Sobolev, K. Yamaoka, J. Zschau, Ann. Geophys. Italy 54, 315 (2011)

47. A. Peresan, V.G. Kossobokov, G.F. Panza, Rend. Lincei-Sci Fis. 23, 131 (2012)

48. I. Kawasaki, Earth Planets Space 56, 813 (2004) 
49. S. Uyeda, T. Nagao, M. Kamogawa, Earthquake precursors and prediction, in Encyclopedia of Solid Earth Geophysics, edited by H.K. Gupta (Springer, Dordrecht, 2011), pp. $168-178$

50. I.P. Dobrovolsky, N.I. Gershenzon, M.B. Gokhberg, Phys. Earth Planet. In. 57, 144 (1989)

51. D. Finkelstein, R.D. Hill, J.R. Powell, J. Geophys. Res. 78, 992 (1973)

52. H. Mizutani, T. Ishido, T. Yokokura, S. Ohnishi, Geophys. Res. Lett. 13, 365 (1976)

53. F.D. Stacey, M.J.S. Johnston, Pure Appl. Geophys. 97, 146 (1972)

54. J. Zlotnicki, F.H. Cornet, J. Geophys. Res. 91, 709 (1986)

55. G. Balasis, M. Mandea, Tectonophysics 431, 173 (2007)

56. D.V. Fitterman, J. Geophys. Res. 84, 6031 (1979)

57. G. Guo, B. Wang, Int. J. Remote Sens. 29, 1921 (2008)

58. Y. Okada, S. Mukai, R.P. Singh, Adv. Space Res. 33, 254 (2004)

59. R.P. Singh, J. Senthil Kumar, J. Zlotnicki, M. Kafatos, Appl. Geochem. 25, 580 (2010)

60. A. Eleftheriou, C. Filizzola, N. Genzano, T. Lacava, M. Lisi, R. Paciello, N. Pergola, F. Vallianatos, V. Tramutoli, Pure Appl. Geophys. 173, 285 (2016)

61. D. Ouzounov, N. Bryant, T. Logan, S. Pulinets, P.Taylor, Phys. Chem. Earth 31, 154 (2006)

62. A.A. Tronin, M. Hayakawa, O.A. Molchanov, J. Geodyn. 33, 519 (2002)

63. F.T. Freund, I.G. Kulahci, G. Cyr, J. Ling, M. Winnick, J. Tregloan-Reed, M.M. Freund, J. Atmos. Sol-Terr. Phy. 71, 1824 (2009)

64. M. Hayakawa, Atmospheric and Ionospheric Electromagnetic Phenomena Associated With Earthquakes (Terra Sci. Publ. Comp., Tokyo, 1999)

65. M. Hayakawa, S.S. Sazhin, Planet. Space Sci. 40, 1325 (1992)

66. V.A. Liperovsky, O.A. Pokhotelov, E.V. Liperovskaya, M. Parrot, C. V. Meister, O.A. Alimov, Surv. Geophys. 21, 449 (2000)

67. S.A. Pulinets, Terr. Atmos. Ocean. Sci. 15, 413 (2004)

68. H.V. Alvan, F.H. Azad, S. Mansor, Adv. Space Res. 52, 1678 (2013)

69. M. Hayakawa, O.A. Molchanov, Phys. Chem. Earth 29, 617 (2004)

70. H.S. Virk, V. Walia, A.K. Sharma, Curr. Sci. 69, 452 (1995)

71. H.S. Virk, B. Singh, Geophys. Res. Lett. 21, 737 (1994)

72. J. Bajc, A. Aoudia, A. Sarao, P. Suhadolc, Geophys. Res. Lett. 28, 1839 (2001)

73. A. Borghi, A. Aoudia, R.E.M. Riva, R. Barzaghi, Tectonophysics 465, 177 (2009)

74. F. Fitzko, P. Suhadolc, A. Aoudia, G.F. Panza, Tectonophysics 404, 77 (2005)

75. E.M. Scordilis, C.B. Papazachos, G.F. Karakaisis, V.G. Karakostas, J. Seismol. 8, 57 (2004)

76. S.B. Yu, L.C. Kuo, Y.J. Hsu, H.H. Su, C.C. Liu, C.S. Hou, J.F. Lee, T.C. Lai, C.C. Liu, C.L. Liu, T. F. Tseng, C. S. Tsai, T.C. Shin, Bull. Seismol. Soc. Am. 91, 995 (2001)

77. A. Bhardwaj, S. Singh, L. Sam, A. Bhardwaj, F. J. Martín-Torres, A. Singh, R. Kumar, Remote Sens. Environ. 188, 1 (2017)

78. A.K. Saraf, V. Rawat, P. Banerjee, S. Choudhury, S.K. Panda, S. Dasgupta, J.D. Das, Nat. Hazards 47, 119 (2008)

79. A.K. Saraf, S. Choudhury, Int. J. Remote Sens. 26, 2705 (2005)

80. V. Tramutoli, V. Cuomob, C. Filizzolab, N. Pergolab, C. Pietrapertosa, Remote Sens. Environ. 96, 409 (2005)

81. K. Cao, Q. Huang, Ann. of GIS, 24, 125 (2018)

82. C. Fidani, F. Freund, R. Grant, Animals 4, 292 (2014)

83. J.L. Kirschvink, Bull. Seismol. Soc. Am. 90, 312 (2000)

84. J.M. Logan, Nature 265, 404 (1997)

85. R.B. Schall, California Geol. 41, 41 (1988)

86. M.D. Barberio, M. Barbieri, A. Billi, C. Doglioni, M . Petitta, Sci. Rep-UK. 7, 11735 (2017)

87. R.K. Chadha, A.P. Pandey, H.J. Kuempel, Geophys. Res. Lett. 30, 642 (2003)

88. G. Martinelli, Seismol. Res. Lett. 71, 583 (2000)

89. E.A. Roeloffs, Pure Appl. Geophys. 126, 177 (1988) 
90. M. Hayakawa, R. Kawate, O.A. Molchanov, K. Yumoto, Geophys. Res. Lett. 23, 241 (1996)

91. Y.A. Kopytenko, T.G. Matishvili, P.M. Voronov, E.A. Kopytenko, O.A. Molchanov, Phys. Earth Planet. In. 77, 85 (1993)

92. F. Freund, Acta Geophys. 58, 719 (2010)

93. F. Freund, A. Takeuchi, B.E.S. Lau, Phys. Chem. Earth 31, 389 (2006)

94. D.V. Fitterman, J. Geophys. Res. 83, 5923 (1978)

95. I. Yamada, K. Masuda, H. Mizutani, Phys. Earth Planet. In. 57, 157 (1989)

96. S.M.Yoshida, M. Uyeshima, M. Nakatani, J. Geophys. Res. 102, 14883 (1997)

97. L. Claesson, A. Skelton, C. Graham, C. Dietl, M. Mörth, P. Torssander, I. Kockum, Geology 32, 641 (2004)

98. T. Mathanlal, Design and Development of Perpetual Environmental Station, Master's Dissertation, Luleå University of Technology, Sweden 2017, http://urn.kb.se/ resolve?urn=urn:nbn: se: ltu: diva -64612

99. F. Freund, J. Geodyn. 33, 543 (2002)

100. F. Freund, J. Sci. Explor. 17, 37 (2003)

101. F. Freund, Nat. Hazards Earth Sys. 7, 543 (2007)

102. L. Piroddi, G. Ranieri, IEEE J. Sel. Top. Appl. 5, 307 (2012)

103. L. Piroddi, G. Ranieri, F. Freund, A. Trogu, Geophys. J. Int. 197, 1532 (2014)

104. R. Petrini, F. Italiano, A. Riggio, F.F. Slejko, M. Santulin, A. Buccianti, P. Bonfanti, D. Slejko, B. Geofis. Teor. Appl. 53, 113 (2012)

105. C. Keller, F.T. Freund, D.P. Cruikshank, Electric properties of water ice doped with hydrogen peroxide (H2O2): implications for icy moons such as Europa (American Geophysical Union, San Francisco, 2012)

106. R.A. Grant, J.P. Raulin, F.T. Freund, Phys. Chem. Earth 85, 69 (2015)

107. I. Wickelgren, IEEE Spectr. 33, 32 (1996)

108. D. Ouzounov, F. Freund, Adv. Space Res. 33, 268 (2004)

109. A.K. Saraf, S. Choudhury, Int. J. Remote Sens. 26, 1065 (2005)

110. M. Blackett, M.J. Wooster, B.D. Malamud, Geophys. Res. Lett. 38, L15303 (2011)

111. G.M. Molchan, Y.Y. Kagan, J. Geophys. Res-Sol. Ea. 97, 4823 (1992)

112. G. Purcaru, Phys. Earth Planet. In. 18, 274 (1979) 